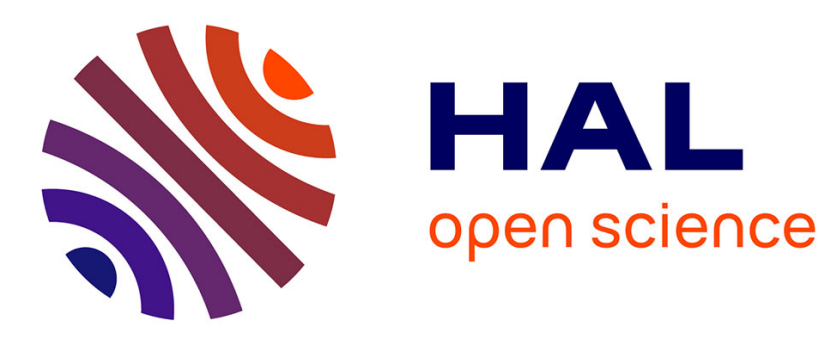

\title{
Towards sensitive, high-throughput, biomolecular assays based on fluorescence lifetime
}

\author{
Anastasia Ioanna Skilitsi, Timothé Turko, Damien Cianfarani, Sophie Barre, \\ Wilfried Uhring, Ulrich Hassiepen, Jérémie Léonard
}

\section{- To cite this version:}

Anastasia Ioanna Skilitsi, Timothé Turko, Damien Cianfarani, Sophie Barre, Wilfried Uhring, et al.. Towards sensitive, high-throughput, biomolecular assays based on fluorescence lifetime. Methods and Applications in Fluorescence, 2017, 5 (3), pp.034002. 10.1088/2050-6120/aa7f66 . hal-02435550

\author{
HAL Id: hal-02435550 \\ https://hal.science/hal-02435550
}

Submitted on 10 Jan 2020

HAL is a multi-disciplinary open access archive for the deposit and dissemination of scientific research documents, whether they are published or not. The documents may come from teaching and research institutions in France or abroad, or from public or private research centers.
L'archive ouverte pluridisciplinaire HAL, est destinée au dépôt et à la diffusion de documents scientifiques de niveau recherche, publiés ou non, émanant des établissements d'enseignement et de recherche français ou étrangers, des laboratoires publics ou privés. 


\title{
Towards Sensitive, High-Throughput, Biomolecular Assays Based on Fluorescence Lifetime
}

\author{
Anastasia Ioanna Skilitsi ${ }^{1}$, Timothé Turko ${ }^{2}$, Damien Cianfarani ${ }^{1}$, Sophie Barre ${ }^{1}$, Wilfried Uhring ${ }^{2}$, Ulrich \\ Hassiepen ${ }^{3}$, Jérémie Léonard ${ }^{*}$
}

1. Université de Strasbourg, CNRS, Institut de Physique et Chimie des Matériaux de Strasbourg, UMR 7504, F67000 Strasbourg, France

2. ICube, UMR 7357, CNRS - Université de Strasbourg, France

3. Novartis Institutes for Biomedical Research, Novartis Pharma AG, 4002 Basel, Switzerland

*Jeremie.Leonard@ipcms.unistra.fr

\begin{abstract}
Time-resolved fluorescence detection for robust sensing of biomolecular interactions is developed by implementing Time Correlated Single Photon Counting in high-throughput conditions. Droplet microfluidics is used as a promising platform for the very fast handling of low-volume samples. We illustrate the potential of this very sensitive and cost-effective technology in the context of an enzymatic activity assay based on fluorescently-labeled biomolecules. Fluorescence lifetime detection by Time Correlated Single Photon Counting is shown to enable reliable discrimination between positive and negative control samples at a throughput as high as several hundred samples per second.
\end{abstract}

Keywords: Time-Resolved Fluorescence, Time Correlated Single Photon Counting, Biomolecular Interaction Assays, High-Throughput Screening, Droplet Microfluidics

\section{Introduction}

Fluorescence detection is a widespread technique used in life sciences and pharmaceutical industry for e.g. wide field microscopy (1-3), flow cytometry $(4,5)$ cell sorting $(6,7)$ or high-throughput screening (HTS) (8-10). Multiple variants of fluorescence-based detection techniques are used for sensing local physico-chemical environment properties or interactions between fluorescently-labeled biomolecules. They include fluorescence intensity (FLINT), fluorescence polarization/anisotropy (FP), single-molecule detection methods or fluorescence lifetime (FLT). Of particular interest, FLT is an intrinsic reporter of the fluorescence quantum yield of a fluorophore, and therefore of the fluorophore interactions with its microenvironment. This means in particular that parameters such as fluorophore concentration, excitation light intensity, excitation light screening or fluorescence reabsorption, which do not modify the fluorophore quantum yield, will not alter the fluorophore FLT. Instead they do alter all the other timeintegrated fluorescence techniques (e.g. FLINT of FP), which makes them less reliable for the accurate 
sensing of fluorophore interactions. This well-known advantage of FLT detection is for instance at the origin of the very successful development of Fluorescence Lifetime Imaging Microscopy (FLIM). (11-14)

In the context of HTS, the same argument has motivated the development of innovative biochemical constructs taking advantage of FLT detection for the efficient sensing of molecular interactions (15-24). However, although the use of FLT for HTS was first explored more than 15 years ago (25-27), it has still not been commonly applied because implementing FLT in high-throughput conditions remains technologically more demanding than intensity-based fluorescence techniques. FLT sensing has long been applied to flow cytometry (28-30) using rapid, frequency-domain, FLT detection techniques which are applicable to high-throughput conditions. Conversely, FLT detection in the time domain by TimeCorrelated Single Photon Counting (TCSPC) is well-known to be the most sensitive and accurate technique also offering the best FLT time resolution. (31) TCSPC may therefore appear to be the preferred approach for FLT-based biosensing, however it requires counting and time-stamping photons one by one, with at maximum one photon detection event per excitation pulse. (32) A fluorescence decay kinetic trace is thus obtained by reconstructing the histogram of the distribution of detection times, after a large number of excitation laser pulses. The method may thus appear relatively slow and was claimed to be inapplicable to high-throughput FLT detection.(33) Alternative time-domain FLT detection techniques have been specifically developed for HTS (33) or flow cytometry (34), but offering reduced sensitivity and time resolution compared to TCSPC. For instance, the so-called Direct Waveform Recording (DWR) technique (33) relies on the analog recording of the fluorescence decay kinetics triggered by single, nanosecond, intense laser shots at a multi-kHz repetition rate. This approach is much less sensitive in essence and its implementation more expensive than TCSPC because it requires much higher average laser power for excitation (i.e. $10 \mathrm{~mW}$ typically). Still, it was already very efficient at enabling FLT detection for HTS (20-23) and demonstrating the significant advantage of FLT $(23,35)$ (i.e. intrinsic reporter of local fluorophore interactions) over fluorescence intensity in this context.

In contrast to the initial motivations for developing e.g. DWR, however, several applications of TCSPC were recently demonstrated for accurate FLT detection under high throughput conditions. (36-38) In particular, using a microfluidic flow cytometer designed for efficient FLT detection by TCSPC at a throughput up to 3000 particles per minute, Nedbal et al. (37) reported a comprehensive series of experiments demonstrating the superiority of FLT over FLINT for biomolecular interaction sensing in individual cells, based on the detection of FRET (Förster Resonant Energy Transfer) between ns-lived chromophores including fluorescent proteins. We instead have implemented TCSPC in microfluidic droplets and investigated the fundamental limitations imposed by the single photon detection technique on the photon acquisition rate and on the achievable signal-to-noise ratio in high throughput conditions. (36) We demonstrated that accurate discrimination between distinct FLTs (i.e. positive and negative control samples in a HTS protocol) is achievable with TCSPC at a droplet throughput exceeding $1 \mathrm{kHz}$. Droplet microfluidics (DmF) is a promising tool for biotechnologies because it lowers the volume of individual samples from $\mu \mathrm{L}$ range per well in microplates down to the 100 -pL range or less in microdroplets and it considerably increases the samples (droplets) manipulation rate (6,39-42). Consequently, DmF provides particularly challenging conditions in terms of throughput and required sensitivity, to assess the performances of TCSPC for high-throughput FLT detection. 
In the present paper we review our latest developments towards demonstrating a high throughput, costefficient TCSPC detection technique. After describing the technical implementation, we in particular illustrate the potential of the approach by demonstrating the feasibility of an enzymatic activity assay relying on the detection of the FLT by TCSPC, in very high-throughput conditions, in microfluidic droplets. The choice of an enzymatic activity assay as a validation for biomolecular interaction sensing was driven by the high biotechnological interest on enzymes (43-46). Beyond, this paradigmatic assay paves the way for the application of TCSPC to high-throughput biomolecular interactions and biotechnologies in general.

\section{Materials and Methods}

\subsection{Microfluidics}

In this work water-in-oil microdroplets are used as small-volume reservoirs that contain the samples of interest and can be manipulated at very high throughput in microfluidic channels. As previously reported (36), we use the conventional "rapid prototyping" technique (47) to manufacture polydimethylsiloxane (PDMS) microfluidic chips. A so-called T-junction is used for droplet generation: three aqueous inlets merge into one channel which intersects orthogonally a fourth channel flowed with immiscible oil (10:1 mixture of perfluorodecalin with $1 \mathrm{H}, 1 \mathrm{H}, 2 \mathrm{H}, 2 \mathrm{H}$-perfluorodecyltrichlorosilane). The mixing of the three aqueous solutions is initiated by the droplet formation and is further accelerated by chaotic advection induced within the droplets by the wiggling shape of the channel after the T-junction (48). The velocity, size and concentration of the droplets are precisely controlled by regulating the flow rates in the aqueous and oil channels using a commercial flow control system (Elveflow, Elvesys, France). Droplets of less than $200 \mathrm{pL}$ volume and $1 \mu \mathrm{M}$ fluorophore or substrate concentration were used in the present work. This represents no more than 200 attomol $\left(10^{-18} \mathrm{~mol}\right)$ of solute per droplet, i.e. per sample.

\subsection{Enzyme activity assay}

To illustrate the efficiency of the proposed FLT detection technology for HTS, we demonstrate the feasibility of an enzymatic activity assay relying on FLT detection. The assay $(15,18,19)$ is designed to evidence the activity of the protease human pancreatic trypsin (from autolyzed human pancreas, Elastin Product Company, Inc., Owensville, MO, USA, catalogue number TR127) on a peptide substrate tagged with the fluorescent probe PT14. Due to the structural flexibility of the peptide the probe undergoes dynamic quenching resulting in a FLT of $7 \mathrm{~ns}$. When the enzyme cleaves the substrate, the probe is released, and its FLT of $14 \mathrm{~ns}$ is restored. Figure 1 displays the kinetics of FLT recovery after mixing $1 \mu \mathrm{M}$ substrate with $1 \mathrm{nM}$ or $10 \mathrm{nM}$ enzyme in PBS. In this work, the fluorescently-labeled substrate was Biosyntan 9385.1 C(PT14)-GFKY-NH2, purchased from Biosyntan (Berlin, Germany). It was, delivered as dry powder, dissolved in DMSO and kept at -20 C. For all experiments it was dissolved in PBS (phosphate buffered saline, $\mathrm{pH}=7.4$ ) and $0.02 \% \mathrm{v} / \mathrm{v}$ TWEEN (Polyethylene glycol sorbitan monolaurate) was added. In the microfluidic droplets, the concentrations of substrate and enzyme were adjusted to $1 \mu \mathrm{M}$ and $1 \mathrm{nM}$, respectively. 


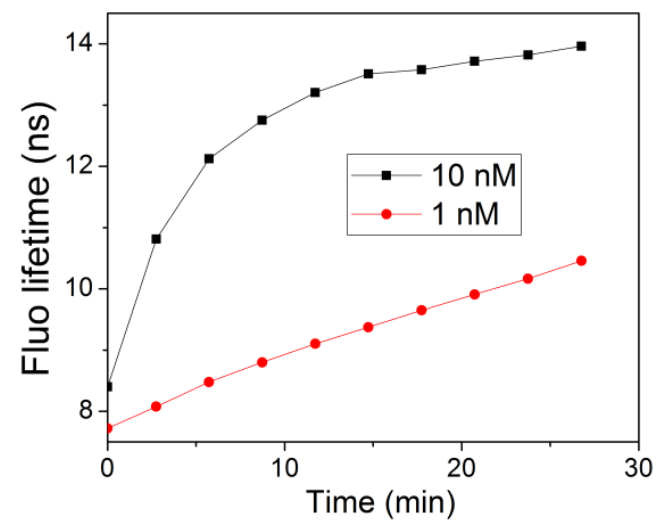

Figure 1: Kinetics of the PT14 FLT recovery upon substrate cleavage in the presence of 1nM or 10nM protease human pancreatic trypsin. Data recorded with a Tecan Ultra Evolution, I20044 microplate reader.

\section{Results}

\subsection{FLT detection set-up}

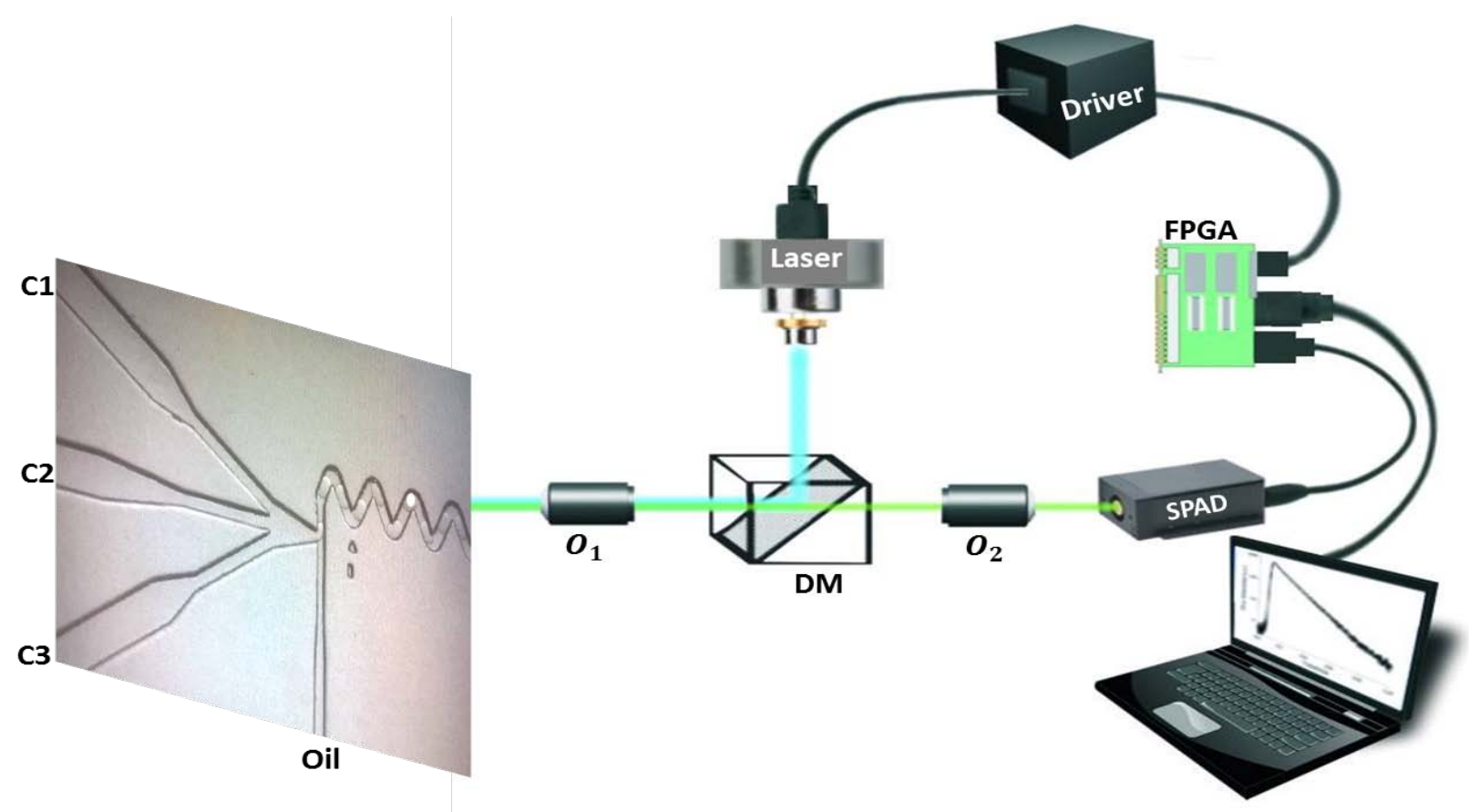

Figure 2. Scheme of the experimental setup. A home-made fluorescence microscope is used to excite and collect fluorescence emission in microfluidic droplets (O1, $\mathrm{O} 2=$ microscope Objectives, $\mathrm{DM}=$ Dichroic Mirror). Water-in-oil droplets are generated in a PDMS microfluidic chip at a so-called T-junction where 3 aqueous channels (C1 to C3) intersect one oil channel. A low-cost TCSPC detection set-up operating at high throughput has been developed, including a cheap diode laser and its home-made driver for pulsed emission (at a $20 \mathrm{MHz}$ repetition rate, $100 \mu \mathrm{W}$ 
average power), a commercial SPAD and a home-made FPGA-based TDC which enables rapid, on-the-fly processing of single photon detection events.

Figure 2 displays a sketch of the experimental set-up. A home-made fluorescence microscope is assembled to detect the fluorescence of water-in-oil droplets circulating in the microfluidic chip. A dichroic mirror (Semrock, BLP01-442R) is used to reflect the excitation light and transmit the fluorescence emission. A microscope objective (Nikon, $x 50, N A=0.6$ ) is used to focus the excitation beam to a $\sim 5$ - $\mu$ m spot inside the droplets circulating in the main microfluidic channel, and to collect and collimate the fluorescence emitted by the droplets. A second objective (Nikon, x20, NA=0.4) is used to focus the fluorescence light on a Single Photon Avalanche Diode (SPAD, ID100, Id-quantique, Switzerland). The excitation source is a diode laser (DL-4146-101S, Roithner lasertechnik, Austria) emitting at $405 \mathrm{~nm}$ and operated in a pulsed mode, by a home-made, cost-effective driver consisting of a current pulse generator described previously. (49) The laser pulse repetition rate is fixed at $20 \mathrm{MHz}$ with 80 ps duration FWHM. The laser average power is 100 $\mu \mathrm{W}$, attenuated to $50 \mu \mathrm{W}$ in the microfluidic experiments performed with the PT14 probe, which is however characterized by a relatively low extinction coefficient $\left(\varepsilon_{\max } \sim 600 \mathrm{M}^{-1} \mathrm{~cm}^{-1}\right.$ at $\left.394 \mathrm{~nm}\right)(15)$.

According to conventional TCSPC experiments, a Time-to-Digital Converter (TDC) is used to measure the time laps between each single photon detection event delivered by the SPAD and the previous laser pulse. As a TDC, we use here a Field-Programmable Gate Array (FPGA, Altera® Cyclone EP4CE55) board configured to realize the function of a TDC. The FPGA board actually generates the $20 \mathrm{MHz}$ clock signal that triggers the laser diode driver, acquires the signal delivered by the SPAD, and measures the time delay between trigger and SPAD signals as a conventional TDC. The design of this home-made, costeffective, accurate FPGA-based TDC was described in detail elsewhere $(50,51)$. It is characterized by a $42-$ ps time resolution, 1280-ns full scale, and 20-ns dead time. As an illustration, Figure 3 displays the fluorescence decay of fluorescein dissolved to $10 \mu \mathrm{M}$ in PBS, acquired in a conventional spectroscopy cuvette with this FPGA-based TDC, and showing the expected monoexponential decay kinetics with a FLT of 4.2 ns.

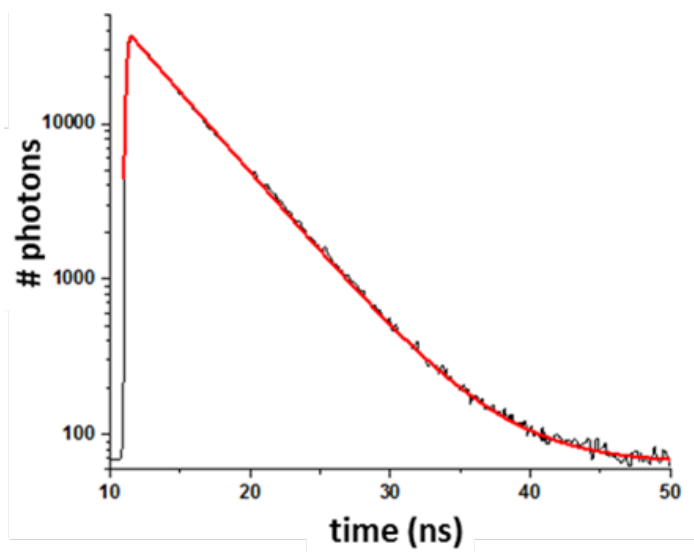

Figure 3: Testing the home-made FPGA-based TCSPC acquisition system. Monoexponential fluorescence decay (semilog plot) of fluorescein in PBS solution measured, with the FPGA-based TDC, in a conventional 1mm-thick quartz cuvette standing in place of the microfluidic chip in the experimental set-up described above. 
The above FPGA-based TDC is used for TCSPC FLT detection in microfluidic droplets as illustrated in Figure 2. A typical experimental run consists of circulating droplets of given size at a controlled flow rate and concentration, while detecting and time-stamping single photons continuously over seconds to minutes. Typical data are displayed in Figure 4. Data processing is performed subsequently as follows. First the analysis of the instantaneous photon counting rate (computed as the number of photons detected

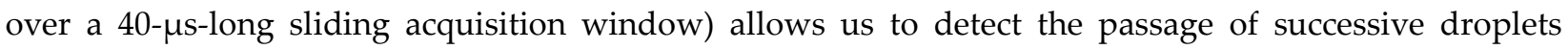
across the excitation laser, with a higher counting rate (i.e. above a fixed threshold) inside droplets, and lower (below threshold) in between two droplets. Second, all the time-correlated, single photon detection events corresponding to a given droplet are assembled in one histogram representing the fluorescence decay kinetics of this very droplet. At a typical $\mathrm{MHz}$ photon counting rate inside the droplets, fluorescence decay curves consisting of a few thousand photons in total may be collected per droplet, at a droplet circulation rate exceeding 300 per second. An automatic fitting procedure is programmed to extract the FLT of all single-droplet histograms, by performing a single parameter monoexponential fit (non-linear least-square minimizing routine implemented with Matlab). More precisely, the fitting function is an exponential decay $(N / \tau \times \exp (t / \tau)$, with $N$ the total number of photons in the histogram, and $\tau$ the FLT), convolved with the instrument response function, determined separately and modeled analytically by a gaussian function of $80 \mathrm{ps}$ full width at half maximum. An example of such a fit is displayed in Figure 4c.

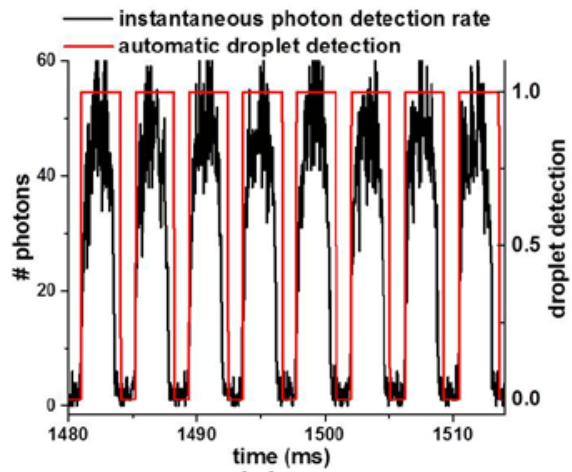

(a)

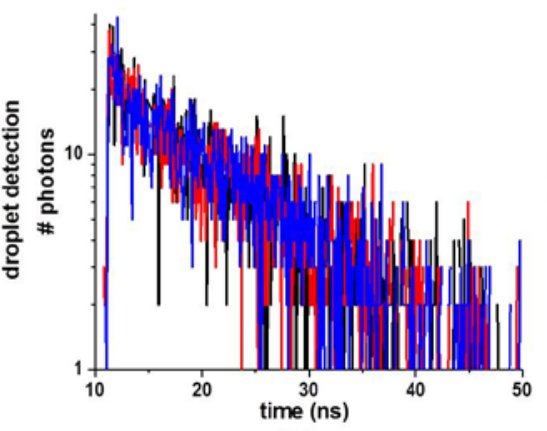

(b)

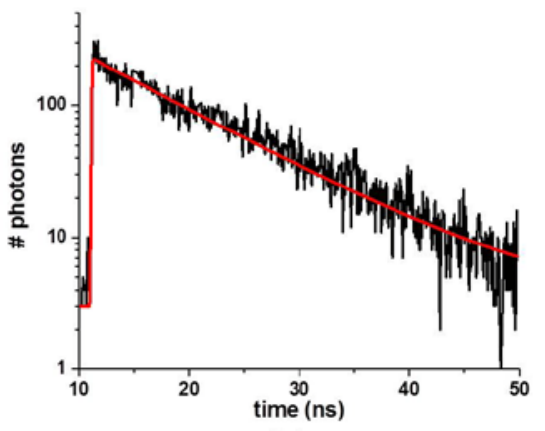

(c)

Figure 4: Implementation of TCSPC detection in microfluidic droplets containing $1 \mu \mathrm{M}$ substrate and $1 \mathrm{nM}$ enzyme and illustration of the data processing. a. Instantaneous photon detection rate as a function of time (black line). The passage of the successive droplets in the focused laser spot is automatically detected (red line). In this example, the fluorescence acquisition time is $\sim 3 \mathrm{~ms}$ per droplet, which is the droplet circulation time across the laser pulse. b. Fluorescence decay histograms of three individual droplets, composed of $\sim 2300$ photons each. c. Sum of the histograms of 10 identical, successive droplets with a mono-exponential fit (red line). This corresponds to a cumulated $30 \mathrm{~ms}$ fluorescence acquisition time, meaning a $\sqrt{10}$ increase in the FLT precision in the case of a monoexponential decay (see text).

Previous investigation of the physical limitations of the photon counting technique in these very high throughput conditions allowed us to make several conclusions (36). First, as a very general property of single photon counting, the measured FLT is accurate as long as the probability that more than one photon hitting simultaneously the SPAD remains low. If this condition is not fulfilled, the measured fluorescence decay histogram is distorted, and the FLT underestimated. This is known as the pile-up effect. $(52,53)$ In the present conditions, it can be shown to remain negligible $(36)$ at photon detection rates 
lower than one tenth of the laser repetition rate typically, i.e. $2 \mathrm{MHz}$ here. Second, due to this fundamental limitation in photon counting rate, the total number of photons detected during a typical $3 \mathrm{~ms}$ exposure time remains relatively small (few thousands), and consequently the photon detection noise (so-called "shot noise") is the origin of the weak signal-to-noise ratio observed in the histograms of individual droplets (Figure $4 \mathrm{~b}$ ). This is therefore also the dominant source of imprecision on the FLT, determined by the monoexponential fit of the noisy histograms. Third and most importantly, this shot noise limits the relative precision on the FLT value to $1 / \sqrt{N}$ at best, with $N$ the total number of photons in the histogram. $(54,55)$ This is a well-known consequence of the Poissonian statistics which characterizes the shot noise inherent to single photon detection. This implies in particular that for a monoexponential decay, measuring a FLT is in principle not less precise and does not require more photons than measuring a fluorescence intensity, since the latter is also characterized by the same $1 / \sqrt{N}$ imprecision at best. With the present experimental set-up, we have shown that the actual relative precision on the measured FLT approaches very closely the ultimate value of $1 / \sqrt{N}$, to within a factor of 1.2 to 1.5 . (36) Therefore accurate discrimination between two distinct FLT's corresponding to positive and negative control samples is achievable in a screening experiment, even at a very high throughput implying the detection of a relatively limited number of photons per sample. This is what we will demonstrate below in a proof of principle experiment based on a biologically relevant, enzyme activity assay.

\subsection{Application to the feasibility of an enzymatic activity assay}

The enzyme activity assay was developed and validated previously $(15,18,19)$. It is based on the increase in the FLT of the PT14 probe which is initially quenched by collisions with the flexible tryrosine residue in the substrate. After trypsin has cleaved the substrate, the tyrosine is separated from PT14 and the probe's FLT is restored. Hence the increase of the FLT is the signature of the enzyme activity. As a test to demonstrate the feasibility of this assay by implementing TCSPC in the conditions illustrated in Figure 3 and 4, we performed two successive experiments, where we measured the FLT of microdroplets circulating at a flow rate of about 300 per second. In the first one, the droplets were produced to contain the substrate solution at $1 \mu \mathrm{M}$ concentration (see Material and Methods), by injecting the same solution in the three aqueous inlets. In the second experiment, a solution of $1 \mu \mathrm{M}$ substrate and $1 \mathrm{nM}$ enzyme was prepared about half an hour in advance such that the enzymatic reaction was initiated (see Figure 1) before injection in the three aqueous inlets of the microfluidic chip. Hence, in these two experiments, each individual droplet is a new control sample to evaluate the FLT of the negative (first experiment) or positive (second experiment) control samples. Each acquisition run lasts for a few seconds only. By analyzing statistically the result of the FLT measurement in each individual droplet (sample), we quantify the precision with which the FLT of the negative and positive control samples may be determined in these very high throughput conditions, and very low sample volumes.

The fluorescence decay histograms obtained for individual droplets were composed of approximately 2300 photons and were fitted automatically as described above, with the FLT $\tau$ as a unique fitting parameter. Figure 5a displays the distribution of the FLT's measured on thousands of droplets in both experiments. More precisely, the FLT of the negative control sample (first experiment) is determined to be $\tau^{-}=6.9 \mathrm{~ns}$ with a RMS standard deviation of $\sigma^{-}=0.25 \mathrm{~ns}$ (green distribution), while that of the positive 
control (second experiment) is $\tau^{+}=11.4 \mathrm{~ns}$ with $\sigma^{+}=0.42 \mathrm{~ns}$ (red distribution). The corresponding coefficients of variations ( $\mathrm{CV}^{\prime} \mathrm{s}$ ) are thus no more than $\sim 3.6 \%$ in both cases. The feasibility of the enzymatic assay in these very high throughput conditions may be quantified by the so-called $Z^{\prime}$ factor (56). In the present case of an assay based on a FLT measurement it writes: $Z^{\prime}=1-\frac{3 \sigma^{+}+3 \sigma^{-}}{\tau^{+}-\tau^{-}}$, with the notations introduced above. With as little as $3 \mathrm{~ms}$ exposure time per sample we already achieve $Z^{\prime}=0.55$, which exceeds 0.5 and therefore demonstrates the feasibility of the assay in these conditions.

Finally, we note that since the precision on the FLT determination is limited by Poisonian photon shot noise(36), a 10 fold increase in the sample exposure time should improve by $\sqrt{10}$ the CV's. This is easily confirmed by summing the histograms of 10 successive droplets (all droplets are identical in a given experiment), before performing the automatic fit of the resulting histograms. The results are displayed in Figure $5 \mathrm{~b}$ and confirm that the $\mathrm{CV}^{\prime}$ 's decrease to $1.2 \%$ as expected. By this analysis, we show that extending the exposure time to $30 \mathrm{~ms}$, i.e. reducing the throughput to 1800 samples per minute which is still very high, the assay can be performed with a $Z^{\prime}$ factor as large as $Z^{\prime}=0.85$.

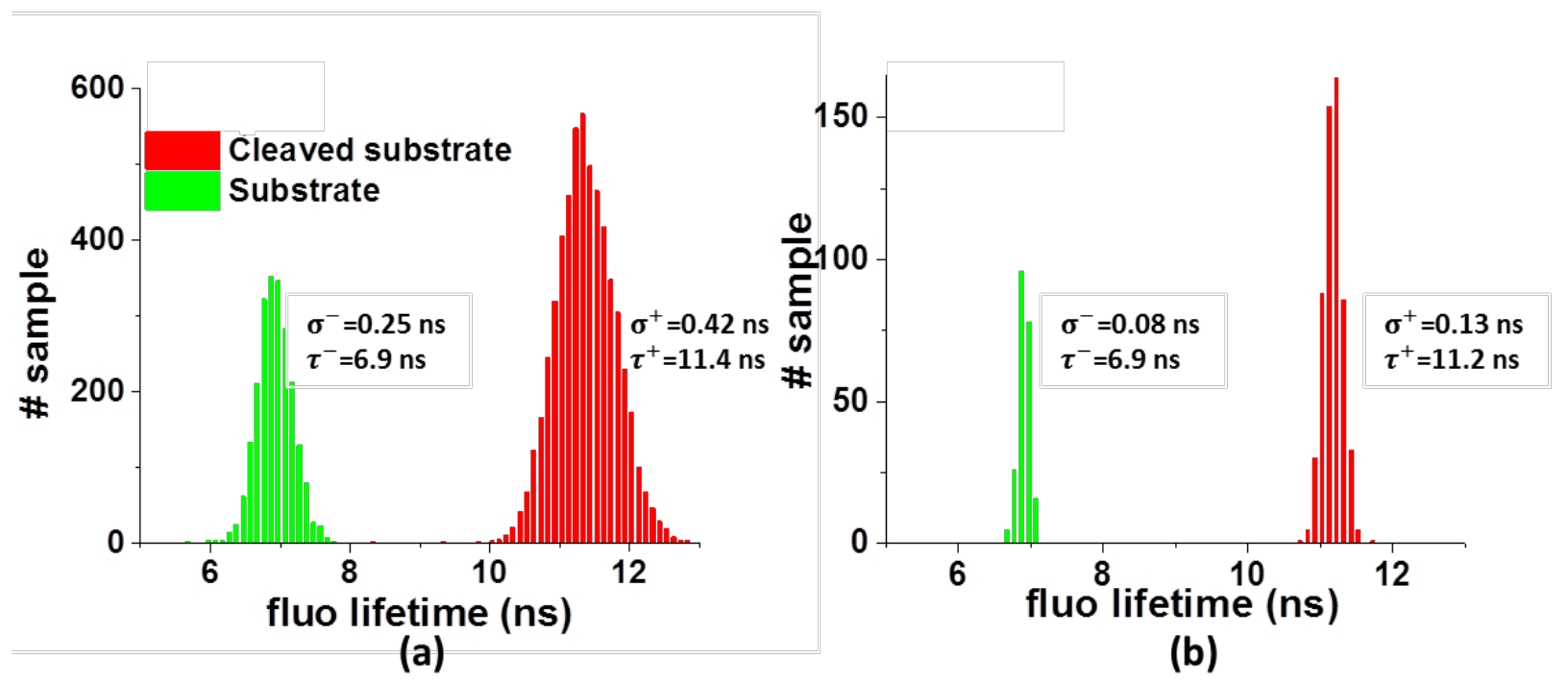

Figure 5: a. Distribution of the FLT measured on individual droplets circulating at a flow rate of 300/s across the excitation laser beam. The droplets contain $1 \mu \mathrm{M}$ substrate with (red) or without (green) addition of $1 \mathrm{nM}$ enzyme. The CVs are $3.6 \%$ resulting in $Z^{\prime}=0.55$ (see text) b. Summing individual droplets histograms 10 by 10 is equivalent to a 10 fold increase of the effective droplets exposure time. Repeating the same statistical analysis on this second set of histograms containing 10 times more photons on average reduces the $C^{\prime} V^{\prime}$ s to $1.2 \%$ and yields $Z^{\prime}=0.85$.

\section{Discussion and Conclusion}

In assessing the performances of TCSPC for high-throughput FLT detection, it is important to remember that in a TCSPC experiment the precision achieved on the measured FLT theoretically scales as $1 / \sqrt{N}$, where $N$ is the total number of photons in the entire histogram (rather than the number of photons in the dominant bin). More precisely (54), it is in principle enough to detect $10^{4}$ photons to achieve a relative precision (i.e. coefficient of variation, CV) of $1 \%$ on the FLT of a monoexponential decaying fluorescent probe. As in our previous work (36), the above results confirm that in a realistic, simple experimental 
implementation (home-made microscope), a CV value of $1.2 \%$ (see Figure $5 b$ ) can easily be achieved by collecting about $2 \times 10^{4}$ photons per histogram (as in Figure 4c) in a cumulated $30 \mathrm{~ms}$ exposure time. This corresponds to an $800 \mathrm{kHz}$ photon counting rate, which is low enough, in the present experiment performed with a $20 \mathrm{MHz}$ laser repetition rate, to prevent any significant pile-up effect that would affect the accuracy of the determined FLT (36).

When comparing the performances of the DWR and TCSPC techniques for high throughput FLT detection, we note that DWR does not perform better in terms of acquisition speed, since $50 \mathrm{~ms}$ are required to achieve $\mathrm{CV}=1.64 \%$ when measuring the $4 \mathrm{~ns}$ FLT of Rhodamine $6 \mathrm{G}$ with the DWR technique (57). Importantly, while the limitation on the FLT precision and its physical origin are well characterized in the case of TCSPC (due to Poissonian photon shot noise, therefore implying the $1 / \sqrt{N}$ scaling law), it is not the case for DWR, where the analog nature of the acquisition scheme introduces additional, electronic sources of noise. Their influence on the FLT precision is not straightforwardly characterized.

In terms of detection sensitivity, the single photon detection technique is certainly unparalleled. It enables detection of much lower sample volumes and molecule quantities, ultimately down to the single molecule level. In the present conditions, we emphasize that, the excitation laser average power is as low as $50 \mu \mathrm{W}$ despite the use of a not very bright PT14 chromophore. This certainly contributes making the TCSPC approach very cost-effective. What is more, considering that the sample volumes are as low as 200-pL, the results above demonstrate that the enzymatic activity assay is feasible with substrate (respectively enzyme) quantities as low as $\sim 200$ attomol (respectively 0.2 attomol) per sample, and at a throughput as high as 300 samples per second. This underlines the extreme sensitivity of TCSPC for FLT determination, even at very high throughput conditions.

Importantly, we stress that a major interest of using droplet microfluidics for the present demonstration is to realize very challenging conditions to assess the performances of TCSPC for very sensitive and accurate FLT sensing at very high throughtput. Certainly the FLT detection technology proposed here and the conclusions of this work would be directly transposable to microplate readers which realize much less challenging conditions in terms of throughput and required sensitivity. Conversely, the present demonstration calls for the implementation of droplet sorting based on FLT detection in DmF chips. This would require on-the-fly processing of single photon detection events in order to (i) detect automatically the passage of successive droplets and (ii) determine the FLT in real time. To the best of our knowledge, no available commercial TDC allows such real-time data processing which requires hardware programming to achieve the desired processing rates. $(38,58)$

In conclusion, the technical implications of the present work are twofold. First it demonstrates that FLT detection is achievable with good precision in very small volume samples and chromophore quantities at high detection throughput. This paves the way to replacing fluorescence intensity by FLT detection for microfluidic applications, including e.g. fluorescence-assisted droplet or cell sorting $(6,59)$. Second it offers a FLT detection which is orders of magnitude more sensitive that DWR and equally (if not more efficiently) applicable to high-throughput acquisition and HTS in particular, also in conventional microplate readers. 
Future development of the TCSPC technique towards improved sensitivity, better FLT precision or shorter exposure times will rely on the ability to increase the maximum photon counting rate. The later is presently limited to the few-MHz range, due to limitations in the SPAD's electrical operation which imposes a typical 40 to 50-ns dead time. However, present CMOS technology enables integrating in a single microelectronic chip multiple, independent channels for time-correlated, single-photon detection in order to parallelize the acquisition of the fluorescence emission of a given sample, so as to enhance significantly the effective photon counting rate. $(60,61)$ We are exploring the implementation of this technology for the present application.

Acknowledgments: This work is supported by the Région Alsace via the Contrat doctoral No. 493-14-C22, the Institut Carnot MICA via the "TR-Fluofluidics" project, and the French Agence Nationale de la Recherche via the ANR-15-CE11-0006 "PICO2" project. The above funders had no role in study design, data collection and analysis, decision to publish, or preparation of the manuscript. The authors have declared that no conflicting interests exist. 


\section{References}

1. Wollman R, Stuurman N. High throughput microscopy: from raw images to discoveries. J Cell Sci. 2007 Oct 9;120(21):3715-22.

2. Pepperkok R, Ellenberg J. High-throughput fluorescence microscopy for systems biology. Nat Rev Mol Cell Biol. 2006;7(9):690-696.

3. Huang B, Bates M, Zhuang X. Super-resolution fluorescence microscopy. Annu Rev Biochem. 2009;78:993-1016.

4. Nebe-von-Caron G, Stephens P., Hewitt C., Powell J., Badley R. Analysis of bacterial function by multi-colour fluorescence flow cytometry and single cell sorting. Microb Anal Single Cell Level. 2000 Sep 1;42(1):97-114.

5. Krutzik PO, Nolan GP. Fluorescent cell barcoding in flow cytometry allows high-throughput drug screening and signaling profiling. Nat Meth. 2006 May;3(5):361-8.

6. Mazutis L, Gilbert J, Ung WL, Weitz DA, Griffiths AD, Heyman JA. Single-cell analysis and sorting using droplet-based microfluidics. Nat Protoc. 2013 Apr 4;8(5):870-91.

7. Ren L, Chen Y, Li P, Mao Z, Huang P-H, Rufo J, et al. A high-throughput acoustic cell sorter. Lab Chip. 2015;15(19):3870-9.

8. Pope AJ, Haupts UM, Moore KJ. Homogeneous fluorescence readouts for miniaturized highthroughput screening: theory and practice. Drug Discov Today. 1999;4(8):350-62.

9. Popp D, Haeupl C, Doering K. Nanosecond fluorescence lifetime analysis-a new dimension for detection in microplates. In: Society for Biomolecular Screening, Danbury, CT. Hague, Netherlands; 2002.

10. Gribbon P, Sewing A. Fluorescence readouts in HTS: no gain without pain? Drug Discov Today. 2003;8(22):1035-1043.

11. Borst JW, Visser AJWG. Fluorescence lifetime imaging microscopy in life sciences. Meas Sci Technol. 2010 Oct 1;21(10):102002.

12. Wallrabe H, Periasamy A. Imaging protein molecules using FRET and FLIM microscopy. Anal Biotechnol. 2005 Feb;16(1):19-27.

13. Levitt JA, Matthews DR, Ameer-Beg SM, Suhling K. Fluorescence lifetime and polarization-resolved imaging in cell biology. Anal Biotechnol. 2009 Feb 1;20(1):28-36.

14. Gerritsen H, Asselbergs $M$, Agronskaia A, Van Sark W. Fluorescence lifetime imaging in scanning microscopes: acquisition speed, photon economy and lifetime resolution. J Microsc. 2002;206(3):218-24.

15. Pritz S, Meder G, Doering K, Drueckes P, Woelcke J, Mayr LM, et al. A Fluorescence Lifetime-Based Assay for Abelson Kinase. J Biomol Screen. 2011 Jan 1;16(1):65-72. 
16. Pritz S, Doering K, Woelcke J, Hassiepen U. Fluorescence lifetime assays: current advances and applications in drug discovery. Expert Opin Drug Discov. 2011 Jun;6(6):663-70.

17. Lebakken CS, Hee Chol Kang, Vogel KW. A Fluorescence Lifetime Based Binding Assay to Characterize Kinase Inhibitors. J Biomol Screen. 2007 May 21;12(6):828-41.

18. Doering K, Meder G, Hinnenberger M, Woelcke J, Mayr LM, Hassiepen U. A Fluorescence LifetimeBased Assay for Protease Inhibitor Profiling on Human Kallikrein 7. J Biomol Screen. 2008 Nov 21;14(1):1-9.

19. Boettcher A, Gradoux N, Lorthiois E, Brandl T, Orain D, Schiering N, et al. Fluorescence LifetimeBased Competitive Binding Assays for Measuring the Binding Potency of Protease Inhibitors In Vitro. J Biomol Screen. 2014;19(6):870-877.

20. Schaaf TM, Peterson KC, Grant BD, Bawaskar P, Yuen S, Li J, et al. High-throughput spectral and lifetime-based FRET screening in living cells to identify small-molecule effectors of SERCA. J Biomol Screen. 2016;1087057116680151.

21. Rebbeck RT, Essawy MM, Nitu FR, Grant BD, Gillispie GD, Thomas DD, et al. High-Throughput Screens to Discover Small-Molecule Modulators of Ryanodine Receptor Calcium Release Channels. J Biomol Screen. 2016 Oct 19;1087057116674312.

22. Petersen KJ, Peterson KC, Muretta JM, Higgins SE, Gillispie GD, Thomas DD. Fluorescence lifetime plate reader: Resolution and precision meet high-throughput. Rev Sci Instrum. 2014 Nov;85(11):113101.

23. Gruber SJ, Cornea RL, Li J, Peterson KC, Schaaf TM, Gillispie GD, et al. Discovery of Enzyme Modulators via High-Throughput Time-Resolved FRET in Living Cells. J Biomol Screen. 2014 Feb;19(2):215-22.

24. Margineanu A, Chan JJ, Kelly DJ, Warren SC, Flatters D, Kumar S, et al. Screening for protein-protein interactions using Förster resonance energy transfer (FRET) and fluorescence lifetime imaging microscopy (FLIM). Sci Rep. 2016 Jun 24;6:28186.

25. Swift KM, Matayoshi ED. High throughput screening using dynamic fluorescence. In: Photonics West'95 [Internet]. International Society for Optics and Photonics; 1995 [cited 2017 Jan 16]. p. 182-189. Available from: http://proceedings.spiedigitallibrary.org/proceeding.aspx?articleid=987595

26. French P. Fluorescence lifetime imaging for drug discovery and disease research. SPIE Newsroom [Internet]. 2008 [cited 2016 Dec 20]; Available from: http://www.spie.org/x25793.xml

27. French TE, Bailey B, Stumbo DP, Modlin DN. Time-resolved fluorometer for high-throughput screening. In: BiOS'99 International Biomedical Optics Symposium [Internet]. International Society for Optics and Photonics; 1999 [cited 2017 Jan 16]. p. 272-280. Available from: http://proceedings.spiedigitallibrary.org/proceeding.aspx?articleid=976988

28. Pinsky BG, Ladasky JJ, Lakowicz JR, Berndt K, Hoffman RA. Phase-resolved fluorescence lifetime measurements for flow cytometry. Cytometry A. 1993;14(2):123-35. 
29. Steinkamp JA, Crissman HA. Resolution of fluorescence signals from cells labeled with fluorochromes having different lifetimes by phase-sensitive flow cytometry. Cytometry A. 1993;14(2):210-6.

30. Houston JP, Naivar MA, Freyer JP. Digital analysis and sorting of fluorescence lifetime by flow cytometry. Cytometry A. 2010;77(9):861-72.

31. Boens N, Qin W, Basarić N, Hofkens J, Ameloot M, Pouget J, et al. Fluorescence lifetime standards for time and frequency domain fluorescence spectroscopy. Anal Chem. 2007;79(5):2137-49.

32. Becker W. Advanced time-correlated single photon counting techniques. Springer Science \& Business Media; 2005. (Springer series in chemical physics; vol. 81).

33. Muretta JM, Kyrychenko A, Ladokhin AS, Kast DJ, Gillispie GD, Thomas DD. High-performance timeresolved fluorescence by direct waveform recording. Rev Sci Instrum. 2010;81(10):103101.

34. Li W, Vacca G, Castillo M, Houston KD, Houston JP. Fluorescence lifetime excitation cytometry by kinetic dithering. Electrophoresis. 2014;35(12-13):1846-54.

35. Cornea RL, Gruber SJ, Lockamy EL, Muretta JM, Jin D, Chen J, et al. High-Throughput FRET Assay Yields Allosteric SERCA Activators. J Biomol Screen. 2013 Jan;18(1):97-107.

36. Léonard J, Dumas N, Caussé J-P, Maillot S, Giannakopoulou N, Barre S, et al. High-throughput timecorrelated single photon counting. Lab Chip. 2014 Aug 15;14(22):4338-43.

37. Nedbal J, Visitkul V, Ortiz-Zapater E, Weitsman G, Chana P, Matthews DR, et al. Time-domain microfluidic fluorescence lifetime flow cytometry for high -throughput Förster resonance energy transfer screening. Cytometry A. 2015;87(2):104-18.

38. Della Rocca FM, Nedbal J, Tyndall D, Krstajić N, Li DD-U, Ameer-Beg SM, et al. Real-time fluorescence lifetime actuation for cell sorting using a CMOS SPAD silicon photomultiplier. Opt Lett. 2016;41(4):673-6.

39. deMello AJ. Control and detection of chemical reactions in microfluidic systems. Nature. 2006 Jul 27;442(7101):394-402.

40. Guo MT, Rotem A, Heyman JA, Weitz DA. Droplet microfluidics for high-throughput biological assays. Lab Chip. 2012;12(12):2146.

41. Miller OJ, El Harrak A, Mangeat T, Baret J-C, Frenz L, El Debs B, et al. High-resolution dose-response screening using droplet-based microfluidics. Proc Natl Acad Sci. 2012;109(2):378-383.

42. Choi J-W, Kang D-K, Park H, deMello AJ, Chang S-I. High-Throughput Analysis of Protein-Protein Interactions in Picoliter-Volume Droplets Using Fluorescence Polarization. Anal Chem. $2012 \mathrm{Apr}$ 17;84(8):3849-54.

43. Cohen P. Protein kinases - the major drug targets of the twenty-first century? Nat Rev Drug Discov. 2002;1(4):309-315. 
44. Turk B. Targeting proteases: successes, failures and future prospects. Nat Rev Drug Discov. 2006 Sep;5(9):785-99.

45. Drag M, Salvesen GS. Emerging principles in protease-based drug discovery. Nat Rev Drug Discov. 2010;9(9):690-701.

46. Jacques $P$, Béchet $M$, Bigan $M$, Caly $D$, Chataigné $G$, Coutte $F$, et al. High-throughput strategies for the discovery and engineering of enzymes for biocatalysis. Bioprocess Biosyst Eng. 2017;40(2):16180.

47. Duffy DC, McDonald JC, Schueller OJA, Whitesides GM. Rapid Prototyping of Microfluidic Systems in Poly(dimethylsiloxane). Anal Chem. 1998 Dec;70(23):4974-84.

48. Song H, Tice JD, Ismagilov RF. A microfluidic system for controlling reaction networks in time. Angew Chem. 2003;115(7):792-796.

49. Uhring $W$, Zint $C-V$, Bartringer J. A low-cost high-repetition-rate picosecond laser diode pulse generator. In: Lenstra D, Morthier G, Erneux T, Pessa M, editors. 2004 [cited 2017 Jan 26]. p. 583. Available from: http://proceedings.spiedigitallibrary.org/proceeding.aspx?doi=10.1117/12.545038

50. Dadouche F, Turko T, Uhring W, Malass I, Dumas N, Le Normand J-P. New Design-methodology of High-performance TDC on a Low Cost FPGA Targets. Sens Transducers. 2015;193(10):123.

51. Dadouche F, Turko T, Malass I, Skilitsi Al, Léonard J, Uhring W. Design, implementation and characterization of Time to Digital Converter on low cost FPGA. In: Yurish S, editor. Advances in Sensors: Reviews, Vol4 "Sensors and Applications in Measuring and Automation Control Systems." IFSA Publishing, S.L.; 2016.

52. Coates PB. The correction for photon pile-up in the measurement of radiative lifetimes. J Phys [E]. 1968;1(8):878.

53. Holzapfel C. On statistics of time-to-amplitude converter systems in photon counting devices. Rev Sci Instrum. 1974 Jul;45(7):894-6.

54. Hall P, Selinger B. Better estimates of exponential decay parameters. J Phys Chem. 1981;85(20):2941-2946.

55. Köllner $M$, Wolfrum J. How many photons are necessary for fluorescence-lifetime measurements? Chem Phys Lett. 1992 Nov 27;200(1-2):199-204.

56. J.-H. Zhang, T. D. Y. Chung, K. R. Oldenburg. A simple statistical parameter for use in evaluation and validation of High Throughput Screening Assays. J Biomol Screen. 1999;(4):67.

57. Petersen KJ, Peterson KC, Muretta JM, Higgins SE, Gillispie GD, Thomas DD. Fluorescence lifetime plate reader: Resolution and precision meet high-throughput. Rev Sci Instrum. 2014 Nov;85(11):113101. 
58. Lieske T, Uhring W, Dumas N, Léonard J, Fey D. Embedded Fluorescence Lifetime Determination for High Throughput Real-Time Droplet Sorting with Microfluidics. In: Conference on Design and Architectures for Signal and Image Processing (DASIP). Dresden, Germany; 2017.

59. Baret J-C, Miller OJ, Taly V, Ryckelynck M, El-Harrak A, Frenz L, et al. Fluorescence-activated droplet sorting (FADS): efficient microfluidic cell sorting based on enzymatic activity. Lab Chip. 2009;9(13):1850.

60. Charbon E. Single-photon imaging in complementary metal oxide semiconductor processes. Philos Trans R Soc Math Phys Eng Sci. 2014 Feb 24;372(2012):20130100-20130100.

61. Krstajić N, Poland S, Levitt J, Walker R, Erdogan A, Ameer-Beg S, et al. 05 billion events per second time correlated single photon counting using CMOS SPAD arrays. Opt Lett. 2015 Sep 15;40(18):4305. 